\title{
ON CERTAIN TWO-POINT EXPANSIONS OF INTEGRAL FUNCTIONS OF EXPONENTIAL TYPE
}

\section{BY I. J. SCHOENBERG}

1. Introduction. Hillel Poritsky* and G. J. Lidstone $\dagger$ found the following formal expansion

$$
f(x)=\sum_{n=0}^{\infty} f^{(2 n)}(1) \Lambda_{n}(x)-\sum_{n=0}^{\infty} f^{(2 n)}(0) \Lambda_{n}(x-1),
$$

where $\Lambda_{n}(x)$ are polynomials (of degree $2 n+1$ ) defined by the generating function

$$
\sinh x t \operatorname{cosech} t=\sum_{n=0}^{\infty} t^{2 n} \Lambda_{n}(x) .
$$

Expansion (1) holds for any polynomial $f(x)$ and solves formally the interpolation problem

$$
f^{(2 n)}(1)=a_{n}, \quad f^{(2 n)}(0)=b_{n}, \quad(n \geqq 0) .
$$

An integral function $f(x)$ is said to be of exponential type if the quantity

$$
\gamma(f)=\varlimsup_{r \rightarrow \infty} \frac{\log M(r)}{r}
$$

which is called the type of the function $f(x)$, is finite, $M(r)$ denoting the maximum modulus of $f(x)$ on the circle $|x|=r$.

Poritsky and J. M. Whittaker $\ddagger$ proved that the expansion (1)

* Hillel Poritsky, On certain polynomial and other approximations to analytic functions, Transactions of this Society, vol. 34 (1932), pp. 274-331.

$\dagger \mathrm{G}$. J. Lidstone, Notes on the extension of Aitken's theorem (for polynomial interpolation) to the Everett types, Proceedings Edinburgh Mathematical Society, vol. 2 (1930), pp. 16-19.

$\ddagger$ J. M. Whittaker, On Lidstone's series and two-point expansions of analytic functions, Proceedings London Mathematical Society, vol. 36 (1933-34), pp. 451-469. In order to facilitate reference we use throughout Whittaker's notations. 
is valid in the finite plane for all integral functions of type

$$
\gamma(f)<\pi .
$$

Whittaker investigated also the interpolation problem

$$
f^{(2 n)}(1)=a_{n}, \quad f^{(2 n+1)}(0)=b_{n}, \quad(n \geqq 0),
$$

which is solved formally by the "generalized Abel expansion"*

(6) $f(x)=\sum_{n=0}^{\infty} f^{(2 n)}(1) M_{n}(x)-\sum_{n=0}^{\infty} f^{(2 n+1)}(0) M_{n+1}^{\prime}(1-x)$,

where the polynomials $M_{n}(x)$ (of degree $2 n$ ) are defined by the generating function

$$
\cosh x t \operatorname{sech} t=\sum_{n=0}^{\infty} t^{n} M_{n}(x) .
$$

Whittaker showed that the expansion (6) is valid in the finite plane for all integral functions of type

$$
\gamma(f)<\frac{\pi}{2} \text {. }
$$

The consideration of the functions $\sin \pi x$ and $\cos \pi x / 2$ shows that $\pi$ and $\pi / 2$ are the best constants on the right sides of the inequalities (4) and (7), respectively, for these functions are respectively solutions of the systems (8) and (10) below.

Our present purpose is to solve the unicity questions connected with the interpolation problems (2) and (5) for functions of exponential type. They are answered completely by the following theorems. $\dagger$

* See W. Goncharoff, Annales École Normale, vol. 47 (1930), pp. 1-78; also I. J. Schoenberg, On the zeros of successive derivatives of integral functions, to appear in the Transactions of this Society.

$\dagger$ It is of interest to mention here a theorem of $F$. Carlson which, in very restricted form, is as follows: An integral function $f(x)$ of type $\gamma(f)<\pi$ which satisfies the system

$$
f(n)=0, \quad(n=0,1,2, \cdots),
$$

must vanish identically (see, for example, E. C. Titchmarsh, Theory of Functions, 1932, p. 186). Here there is no analog to Theorem 1 about the solutions of (C) of exponential type, for $\phi(x)$ sin $\pi x$, with an arbitrary integral function $\phi(x)$, is a solution of (C). Hence such solutions can be of an arbitrary finite type. 
Theorem 1. The only functions of exponential type satisfying the system

$$
f^{(2 n)}(1)=0, \quad f^{(2 n)}(0)=0, \quad(n \geqq 0),
$$

are the finite sine polynomials

$$
f(x)=\sum_{\nu=1}^{k} c_{\nu} \sin \nu \pi x, \quad(\text { of type } k \pi),
$$

with arbitrary constant coefficients $c_{\nu}$.

THEOREM 2. The only functions of exponential type satisfying the system

$$
f^{(2 n)}(1)=0, \quad f^{(2 n+1)}(0)=0, \quad(n \geqq 0),
$$

are the finite cosine polynomials of the form

$$
f(x)=\sum_{\nu=0}^{k} c_{\nu} \cos \frac{(2 \nu+1) \pi}{2} x, \quad(\text { of type }(2 k+1) \pi / 2) .
$$

From Poritsky's and Whittaker's results (4) and (7), it follows only that solutions of (8) and (10) must vanish identically if they are of types less than $\pi$ and $\pi / 2$, respectively.*

2. Proofs of Theorems 1 and 2. We shall use the following lemma of R. D. Carmichael. $\dagger$

Let $f(x)$ be of exponential type not exceeding $\gamma$ and let it have the period $\omega(>0)$. Let $m=[\gamma \omega / 2 \pi]$ denote the greatest integer not exceeding $\gamma \omega / 2 \pi$. Then $f(x)$ has the form

$$
f(x)=\sum_{\nu=-m}^{m} f_{\nu} e^{2 \pi i \nu x / \omega},
$$

where the $f_{\nu}$ 's are constants.

* For a similar extension of a kindred result see A. Weinstein, Zum Phragmén-Lindelöfschen Ideenkreis, Abhandlungen Mathematisches Seminar, Hamburg, vol. 6 (1928), pp. 263-264.

$\dagger$ R. D. Carmichael, Summation of functions of a complex variable, Annals of Mathematics, (2), vol. 34 (1933), pp. 349-378, Theorem 2.4 on p. 362. A proof, omitted by Carmichael, is readily supplied by means of the Fourier expansion of $f(x), f^{(n)}(x)$, their Parseval relations, and Carmichael's Theorem 2.1, p. 361 . 
Let $f(x)$ be a solution of (8) of finite type; then $f(x)$ is necessarily an odd function of period 2. For the equations (8) show that $f(x)$ is odd about the points $x=0$ and $x=1$, whence $f(x)=-f(-x)=f(x+2)$. Applying Carmichael's lemma, for $\omega=2$, we find that $f(x)$ has the form (9), for the cosine terms drop out since $f(x)$ is odd.

To prove Theorem 2 let us assume that $f(x)$ is a solution of (10) of finite type; then $f(x)$ is necessarily an even function of period 4. For the equations (10) show that $f(x)$ is even about the point $x=0$ and odd about the point $x=1$, whence $f(x)=f(-x)=-f(x+2)=-f(-x-2)=f(x+4)$. Carmichael's lemma, for $\omega=4$, shows that $f(x)$ is of the form

$$
f(x)=\sum_{\nu=0}^{m} d_{\nu} \cos \frac{\pi \nu}{2} x
$$

the sine terms having dropped out since $f(x)$ is even. Notice that $f(x)$, as defined by (13), always satisfies the second of the equations (10); it also satisfies the first equation (10) provided

$$
\begin{aligned}
& f^{(2 n)}(1)=(-1)^{n} \pi^{2 n} \sum_{j=0}^{[m / 2]}(-1)^{j} d_{2 j} \cdot j^{2 n}=0, \\
&(n=0,1,2, \cdots) .
\end{aligned}
$$

The first $[m / 2]+1$ equations of the system (14), regarded as a homogeneous system of linear equations in the $d_{2 j}$, suffice already to show that $d_{2 j}=0,(j=0,1, \cdots,[m / 2])$. Hence (13) reduces to the form (11).

3. On Whittaker's Solution of the Interpolation Problem (2). In order to find integral solutions of (2), Whittaker proceeds essentially as follows (loc. cit., Theorem 3, p. 457). Let

$$
h(x)=\sum_{n=0}^{\infty} \frac{a_{n}}{(2 n) !}(x-1)^{2 n}, \quad g(x)=\sum_{n=0}^{\infty} \frac{b_{n}}{(2 n) !} x^{2 n}
$$

and assume that these are integral functions. Let $H(x)$ and $G(x)$ be integral solutions of the difference equations

$$
\begin{gathered}
H(x+2)-H(x)=2 h(x+2), \\
G(x+2)-G(x)=-2 g(x),
\end{gathered}
$$


respectively. Then

$$
f(x)=\frac{1}{2}\{H(x)-H(-x)+G(x)-G(2-x)\}
$$

is an integral function satisfying (1). The proof is immediate, for (15), (16), and (17) imply

$$
f(x)+f(2-x)=2 h(x), \quad f(x)+f(-x)=2 g(x),
$$

and these equations are equivalent with the system (2), in view of (15).

If (2) is to have solutions $f(x)$ of exponential type, then $h(x)$ and $g(x)$ must be of such type, as seen from (18). If

$$
q=\max \{\gamma(h), \gamma(g)\},
$$

it is known that equations (16) have solutions $H(x), G(x)$ of type not exceeding $q$, and then $f(x)$, as defined by (17), is a solution of (2) of type $\gamma(f)=q$. From Theorem 1 we know that all solutions of (2) which are of exponential type are obtained by adding to $f(x)$ an arbitrary sine polynomial of the form (9).

Carmichael has given very convenient expansions for the "principal sums" $H(x)$ and $G(x)$ (loc. cit., Theorem 2.5, p. 365). Introducing these expansions in formula (17), we get a twopoint expansion of $f(x)$ (that is, in terms of $f^{(2 n)}(1)$ and $f^{(2 n)}(0)$ ), provided the type $q$ of $f(x)$ is finite, whereas the PoritskyLidstone expansion (1) breaks down in general if $q \geqq \pi$. Similar remarks apply to the interpolation problem (5).

Swarthmore College 